\title{
Arte para todos. \\ Proyecto de investigación y creación con personas con capacidades diversas
}

\author{
Pablo COCA JIMÉNEZ ${ }^{1}$ \\ Museo Patio Herreriano de Valladolid \\ educacion@museoph.org \\ Alberto OLMOS DE GRACIA ${ }^{2}$ \\ Museo Patio Herreriano de Valladolid \\ albert.2279@yahoo.com \\ Silvia GARCía CEBALlos ${ }^{3}$ \\ MUSEO PATIO HERRERIANO DE VALLADOLID \\ silviart_garciaceballos@hotmail.com \\ Noelia Delgado GonzÁlez, Sonia GARcía VeGAS, Esther SANTIAGO \\ RODRÍGUEZ, Ana Isabel LAGUENS SAEZ ${ }^{4}$
}

Recibido: 01/04/13

Aceptado: 13/10/13

\section{RESUMEN}

Este texto presenta el proyecto Arte para todos, desarrollado por el Museo Patio Herreriano en colaboración el Centro Ocupacional para personas con capacidades diversas del Ayuntamiento de Valladolid. El proyecto tiene como objetivo favorecer la creatividad, la integración social, la autonomía y la autoestima en personas con discapacidad intelectual, a través de procesos creativos relacionados con la creación artística contemporánea.

Palabras clave: Educación artística, arteterapia, capacidades diversas, creación artística contemporánea, creatividad, inclusión social.

\section{Referencia normalizada}

Coca Jiménez, P., Olmos de Gracia, A., García Ceballos, S. ET Al. (2013).

"Arte para todos. Proyecto de investigación y creación con personas con capacidades diversas". En Arteterapia: Papeles de arteterapia y educación artística para la inclusión social Vol.: 8. Páginas 155-168.

\footnotetext{
${ }^{1}$ Coordinador de Educación. Museo Patio Herreriano de Valladolid.

${ }^{2}$ Arteterapeuta.

${ }^{3}$ Arteterapeuta.

${ }^{4}$ Centro Ocupacional para Personas con Discapacidad Intelectual. cindemavallalid@ hotmail.com
} 


\title{
SUMARIO
}

Introducción. El Grupo de trabajo multidisciplinar. Características del colectivo. Planificación del proyecto. Objetivos. Espacios de trabajo. El proyecto como investigación. Intervenciones de carácter creativo. A modo de conclusión.

\section{Art for All. Research and development project with people with diverse abilities}

\begin{abstract}
This paper presents the Art for All project, developed by the Patio Herreriano Museum in collaboration of the Occupational Center for people with diverse abilities of the city council of Valladolid. The project has as aim to encourage creativity, social integration, autonomy and self-esteem in people with intellectual disability through creative processes related to contemporary art.
\end{abstract}

Keywords: Art education, art therapy, diverse abilities, contemporary artistic creation, creativity, social inclusion.

\section{CONTENTS}

Introduction. The Multidisciplinary Working Group. Features of the collective. Project planning. Objectives. Workspaces. The project as research. Interventions creative carácter. In conclusion.

\section{INTRODUCCIÓN}

El proyecto Arte para todos que presentamos a continuación es una iniciativa del Departamento de Investigación y Educación del Museo Patio Herreriano en colaboración con el Centro Ocupacional de personas con discapacidad intelectual, dependiente del Ayuntamiento de Valladolid.

Somos conscientes de que las actuaciones puntuales que habitualmente se realizan en algunos museos con colectivos de personas con capacidades diversas no responden a las características y necesidades de éstos. Después de una profunda reflexión sobre esta cuestión, desde el Museo Patio Herreriano hemos apostado por la realización de actuaciones educativas prolongadas en el tiempo, que se ajusten a las necesidades de este tipo de público, y donde el proceso creativo sea el eje sobre el que pivotan las actividades.

En este sentido, el Museo apuesta cada vez más por proyectos concretos que puedan atender a las características e intereses de colectivos específicos, que doten a estos programas de una mayor continuidad en el tiempo, por lo que las acciones pueden ir adaptándose a las necesidades, tanto del colectivo como del propio proyecto.

\section{EL GRUPO DE TRABAJO MULTIDISCIPLINAR}

Para la definición del proyecto se constituyó un equipo mixto formado por los equipo del Museo Patio Herreriano y del Centro Ocupacional. El primero está 
formado por el responsable del área de educación y por dos colaboradores, estudiantes en prácticas del máster de Arteterapia y educación artística para la inclusión social de la Universidad Complutense, Universidad Autónoma de Madrid, y la Universidad de Valladolid. Mientras, el Centro Ocupacional está integrado por sus cuatro responsables, educadoras especializadas en la educación de personas con discapacidad intelectual.

El carácter multidisciplinar del equipo dota al proyecto de un mayor enriquecimiento aportando diversos puntos de vista, opiniones y experiencias, y posibilita aunar la educación artística y social en un proyecto cuyo objetivo principal es la inclusión social a partir de la práctica artística. Por lo tanto, la cooperación transciende el ámbito institucional asentándose, principalmente, en el espacio de lo profesional.

El proyecto Arte para todos se inscribe en esta línea de proyectos de investigación y colaboración que desde el Museo venimos apostando desde hace algunos años. Este tipo de programas no buscan la ejecución de una actividad, sino que apuestan por un trabajo a largo plazo que posibilite la reflexión crítica respecto al papel del museo en nuestra sociedad, centrándose en públicos en riesgo de exclusión.

Por otro lado, el Centro Ocupacional tiene como prioridad favorecer la inserción socio-laboral, el desarrollo personal y la integración social de las personas que en él participan, prestando una atención de carácter integral y proporcionando apoyos que mejoren sus habilidades adaptativas. Incluir la disciplina artística permite generar un espacio y un tiempo de expresión y reflexión procesual para los participantes, lo que favorece un marco idóneo para el desarrollo de este programa.

El proyecto Arte para todos cuenta con la ayuda de la Fundación DKV Integralia, DKV Seguros y el Hospital Marina de Denia, una institución con una amplia experiencia en programas sobre arte y salud, y que a través del proyecto cuidArt desarrolla en la actualidad un amplio programa de talleres de arteterapia, musicoterapia o arte en vivo con pacientes del Hospital.

\section{CARACTERÍSTICAS DEL COLECTIVO}

El Centro Ocupacional está formado por 54 personas con capacidades diferentes, mayores de edad y con una media de discapacidad de hasta un $65 \%$. En su dinámica diaria el colectivo se distribuyen en cuatro grupos, división que favorece el trabajo y la atención específica, al frente de cada uno de ellos hay una educadora experta en la educación de personas con discapacidad intelectual. Una de las características principales del grupo es su heterogeneidad, tanto desde el punto de vista de la naturaleza de sus discapacidades (procesos genéticos, congénitos, postnatales o ambientales), como respecto a su capacidad autónoma y cognitiva. Además, algunos poseen trastornos sensoriales u obsesivos que se solapan con la discapacidad que padecen. El Centro desarrolla una labor ocupacional mediante 
la elaboración de diferentes objetos que favorecen en sus integrantes el desarrollo de la autonomía, la autoestima y su capacitación e inserción en el mundo laboral.

Respecto a la diversidad del grado de discapacidad que presentan las personas que integran el colectivo se han tomado como modelo dos escalas de clasificación:

Para la American Psychiatric Association (en adelante APA), en su última edición, el DSM-IV-TR ${ }^{5}$ (2002), la cual establece como criterios no sólo el CI, significativamente inferior al promedio, sino que también analiza el déficit o las alteraciones de ocurrencia en la actividad adaptativa en el menos dos de las siguientes áreas: comunicación personal, vida doméstica, habilidades sociales/interpersonales, utilización de recursos comunitarios, autocontrol, habilidades académicas funcionales, trabajo, ocio, salud y seguridad (Castejón, 2008:257). La clasificación establece como referencia no el término discapacidad mental, sino el término "retraso mental", lo cual refleja que pese a que los valores sirvan de referencia, aún se mantiene una nomenclatura que en nada ayuda a la inserción social de personas con capacidades diversas.

Según esta clasificación, el retraso mental medio/moderado determinaría un CI entre 35/40 y 50/55, lo que correspondería con una categoría pedagógica "entrenable", no pudiéndose beneficiarse de los programas educativos existentes. Mientras, el retraso mental leve sitúa el CI entre 50/55 y 70, lo cual correspondería a la categoría pedagógica de "educable" (Verdugo, 1989:50).

En la actualidad, estas escalas están en proceso de transformación, tal y como nos apuntan $\mathrm{M}^{\mathrm{a}}$ José Rabazo Méndez y Juan Manuel Moreno Manso (2007), atrás quedaron los tiempos en que la medición del CI podría medir si un sujeto era o no "entrenable".

De hecho, la AAMR elimina las anteriores categorías para establecer un nuevo modelo de categorización de los apoyos requeridos y no de los individuos. AAMR plantea que "Los apoyos son recursos y estrategias que pretenden promover el desarrollo, educación, intereses y bienestar personal". (Moreno y Montero, 2007:23).

Las tres áreas que se suelen ver afectadas o limitadas en el caso de la discapacidad intelectual son:

Inteligencia conceptual, la cual se refiere al desarrollo de capacidades mentales tales como el aprendizaje, percepción, memoria, elaboración de conceptos, procesamiento de información, análisis, síntesis...

Inteligencia práctica, que incide en la capacidad de autonomía en el cuidado de uno mismo, de independencia en la realización de actividades

\footnotetext{
${ }^{5}$ Diagnostic and Statistical Manual of Mental Disorders
} 
cotidianas como el aseo, el descanso, el mantenimiento alimenticio, la previsión de situaciones como peligro o lesiones, el trabajo, ocio...

Inteligencia social, que engloba los comportamientos sociales de desarrollo típico así como las conductas, los juicios, los comportamientos adecuados en contextos sociales, capacidad de expresión tanto emotiva, afectiva y de pensamientos, comprensión social, intuición, resolución de conflictos, ejecución de juicios éticos,...

Sobre éstas se trabaja en el centro de forma continua, y cuyo proyecto apoyará incidiendo en este último aspecto, la inteligencia social, donde se ha detectado mayor dificultad.

\section{PLANIFICACIÓN DEL PROYECTO}

El proyecto se ha planificado para ser desarrollado a lo largo de un año de trabajo mediante fases consecutivas. Cada periodo forma parte de un mismo proceso que nació en el mes de octubre de 2012 y que finalizará con las exposiciones de las obras generadas en los talleres creativos. No obstante, antes de comenzar el trabajo directamente con los grupos se realizaron diversas reuniones entre los profesionales de ambas instituciones con el fin de poner las bases del proyecto. El intercambio de experiencias y puntos de vista fue muy enriquecedor para generar un programa que pudiera atender a la diversidad propia del colectivo, desde una perspectiva específica de los diferentes casos. Estos encuentros previos generaron además una mayor complicidad entre todos los miembros del equipo de trabajo, propiciando un ambiente rico en propuestas y expectativas.

En este sentido, se estableció como herramienta de trabajo un sistema de reuniones periódicas, tanto en el Museo como en el Centro Ocupacional, con el fin de establecer los objetivos que articularan el proyecto, detectar necesidades, definir las metodologías a seguir en cada fase del proyecto, debatir propuestas y contenidos, decidir criterios de registro y temporalización, etc.

Posteriormente se inició un periodo de observación de los grupos, tanto en su trabajo diario como en aquellas actividades desarrolladas fuera del centro. Este proceso permitió al equipo del Museo conocer las diferentes especificidades y el contexto natural del colectivo. La observación, imprescindible para cualquier proyecto de investigación, es fundamental para conocer las características de las personas que van a centralizar el proyecto, sus comportamientos, actitudes y aptitudes, en definitiva, un conocimiento previo que sin lugar a dudas es necesario.

Finalmente, se estableció una fase de documentación teórica y práctica. Se realizó un amplio rastreo de bases documentales de artículos, proyectos e investigaciones realizadas con personas con capacidades diversas, procesos de intervención y actuación, con el fin de conocer experiencias previas y poder conformar el 
proyecto con los mayores datos posibles, contrastar información, justificar modos de actuación y definir los objetivos que iban a articular el programa educativo.

De este modo, el proyecto Arte para todos se articuló en cuatro fases consecutivas. Una primera, basada en un proceso de indagación sobre las necesidades del grupo resultado de las reuniones, del periodo de observación y documentación de experiencias previas. Una segunda fase basada en las intervenciones de carácter creativo que se desarrollaría en el propio Centro Ocupacional. Una tercera etapa que se realizaría entre los meses de marzo y junio en el Museo, en torno a la exposición Diálogos, y basada en el trabajo directo con las obras de la muestra. Y, finalmente, una cuarta y última fase que supondrá la exposición de las piezas generadas en los talleres del museo, tanto en el Patio Herreriano de Valladolid, como en el Hospital de Denia y en la Fundación Integralia de Barcelona.

El equipo de trabajo decidió registrar minuciosamente todo el proceso. La documentación gráfica (fotografía y vídeo) y textual (actas de reuniones y diarios de las actividades), nos permitiría formalizar reflexiones periódicas sobre la marcha del proyecto, así como desarrollar al final del mismo una revisión crítica de las propuestas elaboradas y de la consecución de los objetivos. Desde nuestro punto de vista es imprescindible documentar todo, cualquier aspecto que aparentemente sea ingenuo o superficial nos puede proporcionar una perspectiva singular y específica del proceso de trabajo.

\section{OBJETIVOS}

Todo proyecto educativo debe estar articulado por unos objetivos que permitan conseguir unas metas o resultados que deseamos conseguir, que permiten articular la selección y organización de los contenidos, y sobre los que basaremos la evaluación final del trabajo (Gimeno, 1989:257).

Una vez realizado el diagnóstico de las características del grupo y de las necesidades de sus integrantes, después de varias sesiones de debate y de observación se establecieron un conjunto de objetivos para desarrollar a lo largo del proyecto.

- Desarrollar la empatía y la autoestima, como complemento para el bienestar de personas con capacidades diversas.

- Regular las conductas para el desarrollo de habilidades sociales y resolución de conflictos, por medio del arte contemporáneo.

- Comprender el espacio museístico como lugar para el desarrollo de aptitudes artísticas y el fomento de la inclusión social.

- Favorecer el desarrollo de actitudes creativas en los participantes. 


\section{ESPACIOS DE TRABAJO}

Aún siendo conscientes de la necesidad de que el proyecto se desarrolle en un espacio artístico, el equipo de trabajo decidió iniciar su labor en el propio Centro Ocupacional, con el fin de comenzar el proceso creativo en el espacio natural del colectivo, para, en un segundo momento, continuar el trabajo en el Museo. Por otro lado, el Centro posee connotaciones plásticas por cuanto parte de la labor ocupacional de estas personas tiene que ver con la elaboración de objetos artesanales. Iniciar las intervenciones en el contexto laboral y familiar del colectivo es fundamental para que el proyecto supusiera una continuidad dentro del trabajo del grupo, aún dentro de la excepcionalidad del proyecto.

Las primeras dinámicas nos permitieron tener un mayor conocimiento de los miembros del colectivo, de sus actitudes frente a la creación artística, de sus habilidades insterpersonales, de sus capacidades y autonomía en la toma de decisiones, así como de sus habilidades respecto a la utilización de herramientas plásticas como la pintura, la fotografía, el vídeo, etc.

Estas primeras actividades han sido fundamentales para establecer una sólida base para trabajar en un segundo momento delante de las obras. Con el fin de concretar un contexto de trabajo específico, se decidió trabajar en torno a la exposición Diálogos, una muestra fundamentada en el diálogo entre la Colección del Museo Patio Herreriano y de la Colección de DKV Seguros, cuyo eje conceptual se basa en la intersección entre arte y salud.

Por otro lado, gracias a la colaboración de la Fundación DKV Integralia, DKV Seguros y el Hospital Marina de Denia, no solo ha permitido que este proyecto sea posible en el actual marco de crisis económica que el país vive, sino que además permitirá que, como resultado del proyecto educativo se pueda realizar una exposición itinerante con las obras generadas a partir de los talleres del museo en torno a la exposición. La muestra, se podrá visitar en tres sedes diferentes: el Museo Patio Herreriano, dentro del proyecto expositivo Sala Cero, el Hospital de Denia y la Fundación Integralia de Barcelona.

\section{EL PROYECTO COMO INVESTIGACIÓN}

El proyecto Arte para todos se definió desde un primer momento como un programa de investigación basado en el procedimiento de comprobar cómo la creación artística contemporánea, en sus diferentes vertientes, es capaz de favorecer, en personas con discapacidad intelectual, el desarrollo de aspectos tales como la autonomía, la creatividad, la autoestima, la empatía o las relaciones interpersonales.

Para este fin, la metodología más adecuada es la Investigación Acción (en adelante I-A). Este método introducido por primera vez por Lewin en 1946, y desarrollado posteriormente por Kolb en 1984 y por Carr y Kemis en 1988 (Latorre, 2003:32), implica una búsqueda de mejora de los objetivos estipulados por parte de los participantes dando lugar a un conjunto de espirales cíclicas de 
planeamiento, acción, observación y reflexión (Escribano, 2004). Este tipo de metodología propone entre sus fines favorecer el desarrollo de la práctica educacional y social, lo que se adecua perfectamente a nuestro objetivo de trabajo. Además, la I-A se caracteriza por poseer un carácter participativo y colaborativo en los usuarios, que deben generar reflexiones constantes sobre sus prácticas habituales. Para ello, según Latorre (2003:28), se desarrolla un plan de acción que pretende comprender e interpretar las situaciones (indagación) para cambiarlas (acción) y mejorarlas (propósito).

Por este motivo, el proyecto propone actividades que nos permitan dar lugar a dinámicas posteriores de reflexión, buscando la comparativa entre la práctica realizada y los conflictos o situaciones incómodas que se dan a lo largo de su día a día. De este modo el grupo de participantes reflexiona acerca de los aspectos que se proponen y nosotros, como educadores, realizamos una revisión constante de las prácticas y las sesiones siempre concluyendo con un compromiso final de mejora.

Según Bisquerra (2000:279), la I-A pretende resolver un problema real y concreto que se establece en un contexto espacio-temporal, sin ánimo de realizar generalizaciones, lo que requiere un conocimiento previo del problema ante cada centro, institución y colectivo con el cual trabajar. Los parámetros que establece este tipo de metodología se enmarcan perfectamente en el objetivo general de este proyecto, que reside en el fomento de la inclusión social trabajado con diversos colectivos y en diferentes ámbitos.

Dentro de las modalidades que existen dentro de la I-A, la que mejor se adecua a nuestro diseño metodológico es la de tipo emancipatorio, ya que el rol de investigador y la relación entre el facilitador y los participantes, es más acorde a nuestra labor como arteterapeutas, o en este caso, como educadores artísticos, moderadores del proceso.

\section{INTERVENCIONES DE CARÁCTER CREATIVO}

La segunda fase consistió en las intervenciones realizadas en el Centro Ocupacional. Algunas de las mayores dificultades que presentaban ciertas personas del colectivo fueron la incapacidad para regular algunas conductas como la afectividad, la empatía, las situaciones de exaltación gestual o emotiva contradictoria, los temperamentos impulsivos o la comprensión de los roles, aspectos que por otro lado, influyen en la convivencia social.

No obstante, las rutinas diarias y los ritmos marcados en su producción laboral se convierten, por otro lado, en un factor potencial para trabajar desde la actividad artística estos déficits. En este sentido, sus actitudes frente a este tipo de tareas, el deseo ferviente por experimentar nuevas propuestas, el afán por alcanzar logros tras finalizar la realización de determinados productos, la curiosidad por realizar trabajos que estimulen su lado creativo, el interés por tomar 
decisiones autónomas o el deseo por romper con la rutina diaria mostrando una gran actitud hacia nuevos aprendizajes, facilitan el desarrollo del proyecto.

Es necesario señalar que el Centro Ocupacional tiene entre sus actividades fuera del área de trabajo habitual, visitar periódicamente museos, salas de exposiciones y centros culturales, lo que les ha proporcionado una base para poder trabajar los déficits antes señalados desde una perspectiva artística.

Como hemos comentado anteriormente, el colectivo de 54 personas se divide a su vez en 4 grupos, de 13 ó 14 personas cada uno. Esta división obedece a cuestiones de operatividad, lo cual favorecía el desarrollo del proyecto.

La primera intervención práctica, realizada en noviembre y diciembre de 2012 en el centro se basó en un juego de imágenes artísticas que los integrantes debían reinterpretar desde sus perspectivas. El grupo es profundamente comunicativo, sus aportaciones son muy sugerentes y encierran habitualmente, reflexiones conscientes o inconscientes, de sus situaciones personales. Las imágenes consistían en un conjunto de obras de arte, realizadas en diferentes medios, y donde se abordaba, desde diferentes perspectivas, la exclusión social.

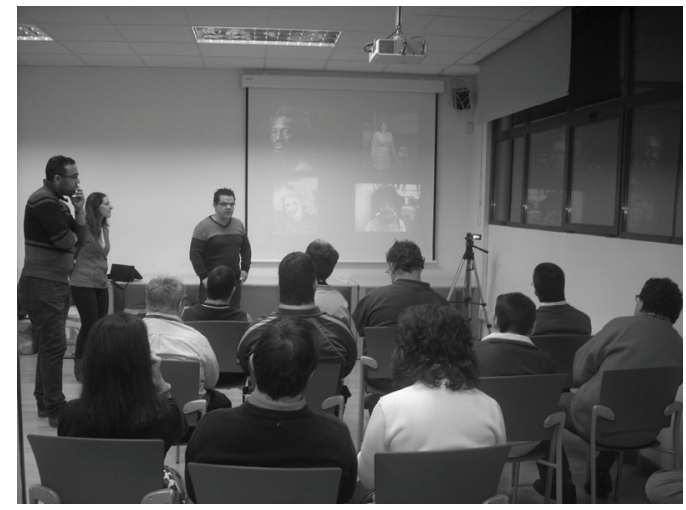

Fig.1 (Primera intervención)

Durante el desarrollo de la actividad, observamos cómo la cuestión de qué es o qué no es arte estaba profundamente arraigada en el grupo. Muchos de las personas asimilaban cualquier obra como parte de las manifestaciones artísticas, mientras otras mostraron una gran resistencia ante la creación artística contemporánea. No obstante, las aportaciones más significativas fueron las reflexiones que algunos extrajeron de las obras en relación a su discapacidad y cómo esta se manifiesta en las miradas de los individuos que les observan, miradas incómodas que les acompañan diariamente. Finalmente se concluyó con un propósito de cambio de mirada de ellos hacia la sociedad y de la sociedad hacia ellos. Se apostó por la transformación o regulación de ciertos aspectos que favorecieran ese cambio de mirada. 
En enero de 2013 se realizó una segunda intervención centrada en la realización de un collage de carácter plástico. El objetivo era generar conflictos de espacio y trabajo que pudieran reproducir sus propios conflictos respecto a las cuestiones de empatía, relaciones interpersonales y emociones. La actividad consistió en rellenar los espacios de una obra abstracta mediante diferentes tipos de materiales. La pieza sobre la que se iba a generar el collage establecía diferentes superposiciones entre los diferentes espacios generando así dificultades de ejecución. El fin de la intervención era detectar estos conflictos para posteriormente reflexionar en grupo sobre ellos. En este tipo de acciones se evidencian las diversidad de actitudes y aptitudes, resistencias, etc.

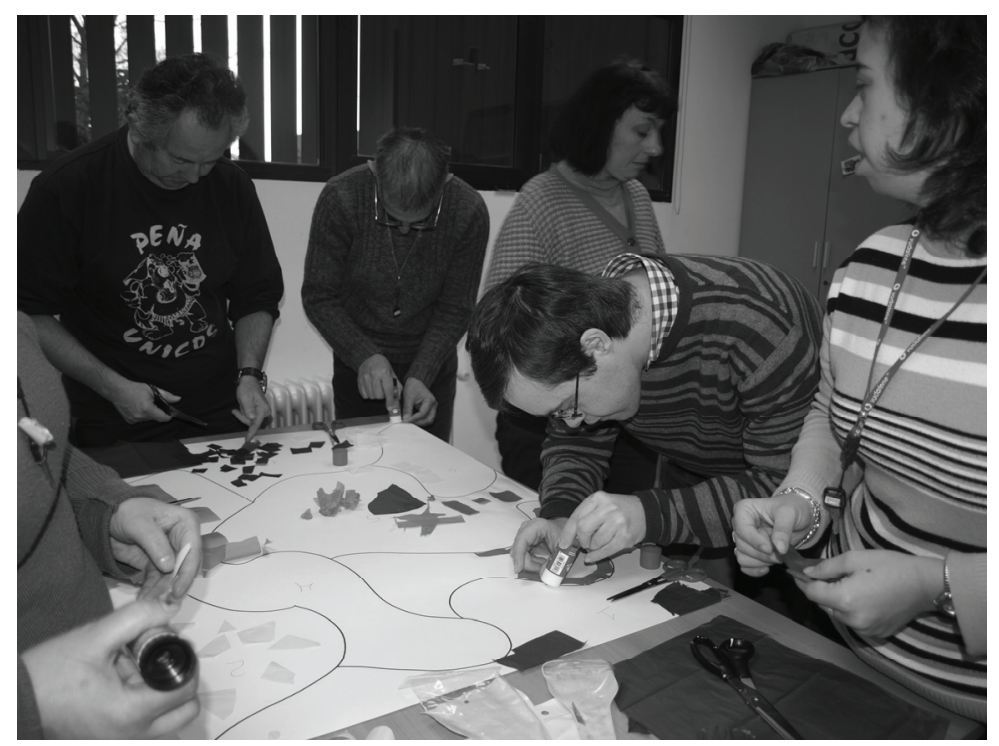

Fig.2 (Segunda intervención. Taller)

A continuación se realizó una reflexión en torno a la actividad que pretendía evidenciar las fisuras propias provocadas por la interacción del grupo, así como hacer surgir posibles conflictos respecto a las cuestiones marcadas. Aunque casi ninguno señaló problema alguno respecto al trabajo en equipo, si hubo algún caso que destacó algún comportamiento inadecuado. Es cierto, que este tipo de dinámicas hace aflorar algunos conflictos entre compañeros. 
Fig.3 (Segunda intervención. Debate reflexivo)

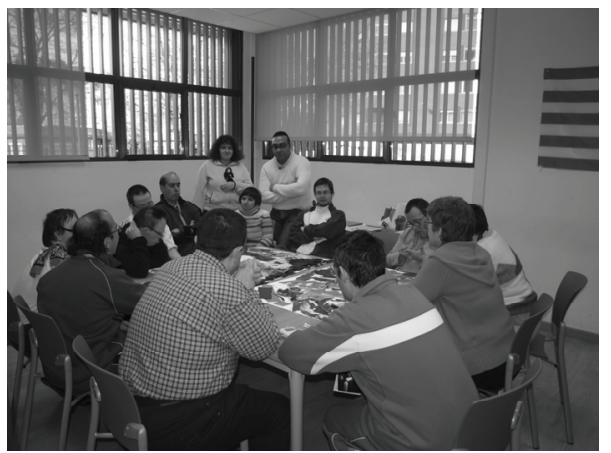

La tercera intervención, desarrollada en febrero de 2013, pretendía que los integrantes del Centro Ocupacional presentaran a través del vídeo y la fotografía su propio contexto laboral. En esta ocasión la actividad implicaba la planificación y autogestión de la presentación y la grabación de la misma, lo que suponía un reto nunca antes experimentado. En este sentido, la intervención tuvo dos fases diferentes. La primera consistió en que cada persona retratara su centro a través de una imagen fotográfica. Ésta debería resumir aquellos aspectos que desde una perspectiva personal, fueran significativas para ellos. En todo momento ellos debían seleccionar aquello que quisiesen fotografiar. Por otro lado, esta acción les permitió experimentar con un medio artístico de una forma libre y autónoma.

Las educadoras del centro señalaron que una de las aportaciones más grandes de esta actividad fue fomentar la autoestima, aspecto que se refleja de forma repetitiva en muchas de las fotos, gracias a que las imágenes eran elaboradas y gestionadas por ellos mismos, sin ningún tipo de intermediación.

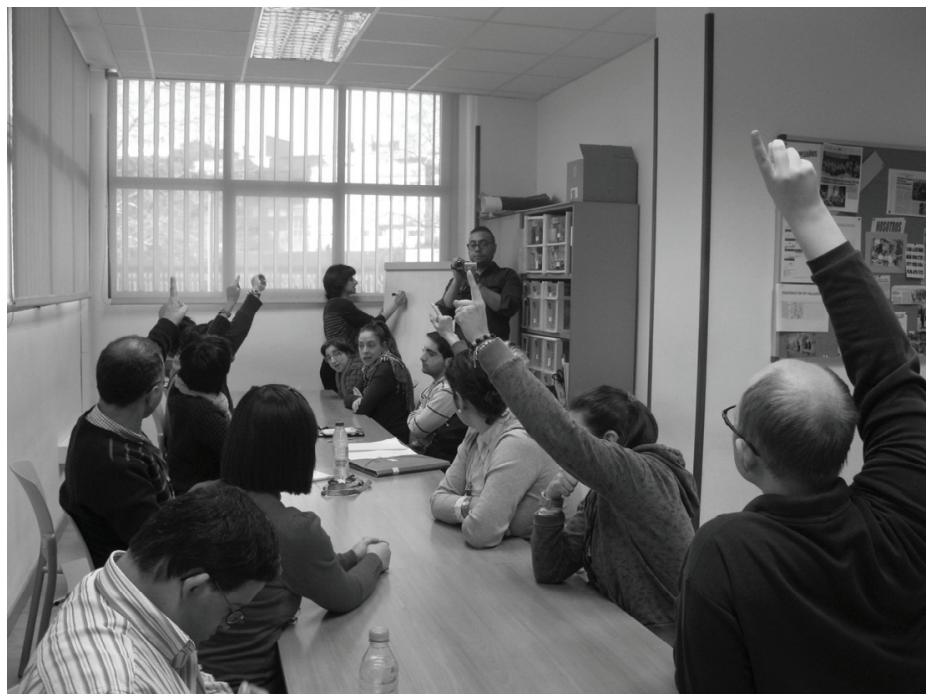

Fig.4

(Tercera intervención. Instante de la reunión para definir la presentación de su Centro) 
Mientras, la segunda parte de esta intervención en torno a la imagen, consistió en presentar su centro a través del vídeo. Para ello, sus integrantes debían organizarse y, con la ayuda de las educadoras del centro, gestionar un guión y grabar aquellos aspectos que querían destacar en la presentación. Para ello, se realizaron reuniones previas para determinar los aspectos querían destacar: las actividades cotidianas, sus impresiones respecto a cómo trabajan, cómo se relacionan, porqué están en este centro, etc. Se decidió en consenso las personas que realizarían la grabación y quienes la presentación.

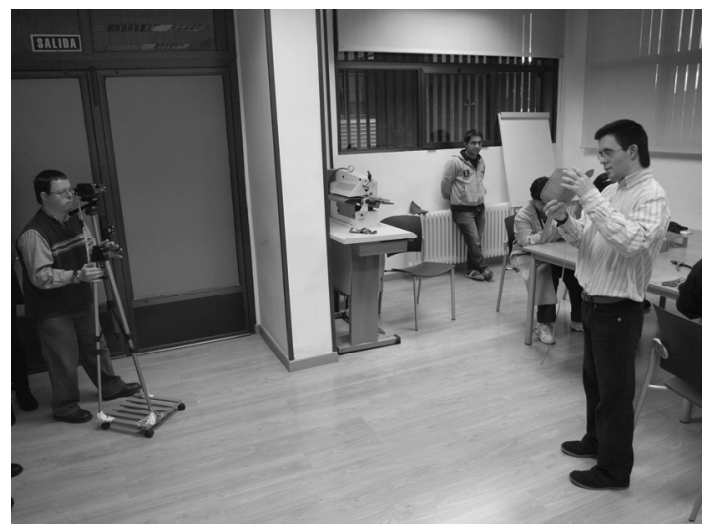

Fig.5 (Tercera intervención. Grabación de la presentación del Centro)

En la actualidad, el proyecto acaba de comenzar su desarrollo en el Museo Patio Herreriano. Después de cinco meses trabajando en el Centro Ocupacional, los grupos han comenzado a visitar el centro de arte y la exposición Diálogos.

\section{A MODO DE CONCLUSIÓN}

Aunque es pronto para realizar una reflexión final sobre el proceso de trabajo, sí podemos adelantar que las actividades generadas hasta la fecha están profundizando en aspectos como la empatía, la afectividad, la creatividad o las relaciones interpersonales.

Los medios utilizados en todo momento están directamente relacionados con las prácticas artísticas contemporáneas. No obstante, la perspectiva educativa y social debe ser el eje fundamental sobre el que pivota el proyecto, y no la actividad artística en sí, aunque este componente sea fundamental. Se continúan explorando las posibilidades de cambio en el desarrollo personal trabajando de forma minuciosa en la observación y recogida de datos de los procesos que se acontezcan en la realización de producciones artísticas.

Toda actividad debe formar parte del proceso creativo, de hecho es el proceso el que sustenta el proyecto de investigación que aúna el esfuerzo de tantas personas. Cada intervención es completada con una reflexión final que permite detectar las fisuras del proyecto, subsanarlas, extraer las conclusiones pertinentes a fin 
de continuar las siguientes fases del programa educativo. Sin un tiempo reservado para la reflexión y un espacio reservado para la creatividad, las posibilidades de crecimiento en las personas, de autorrealizarse y evolucionar, quedarían limitadas.

Por otra parte, empoderar las voces de forma polifónica en este grupo de personas con capacidades diversas es un hecho potenciador, ya que contribuimos a un cambio de mirada tan necesario y por el cual luchan los colectivos en riesgo de exclusión social. Estas son algunas de las razones que nos motivan a seguir brindándoles herramientas por medio del arte, a través del departamento de Investigación y Educación del Museo de Patio Herreriano y del Centro Ocupacional de Valladolid.

Finalmente se ha consolidado un grupo de trabajo que colabora en la investigación, el planteamiento, la estructura y el desarrollo del mismo. Consideramos que este aspecto ha sido muy positivo y fructífero a la hora de realizar el proyecto ya que está resultado ser mucho más enriquecedor y ambicioso de lo que se planteaba en las expectativas iniciales. Cada miembro ha aportado conocimientos al grupo y a su vez ha tomado nuevos aprendizajes de los compañeros que servirán de ayuda en sus experiencias futuras.

Inicialmente fue difícil establecer los límites del proyecto al enfrentarnos a un nuevo colectivo el cual se ha de conocer en profundidad para concretar qué se puede abordar con él y simplificar los objetivos de trabajo. Somos conscientes de que cualquier pequeño cambio es un gran paso en nuestra intervención y por encima de todo se halla el proceso para conseguirlo, sobre el que se reflexiona de forma continua con los participantes y entre el grupo de trabajo.

Por último destacar que los resultados de las intervenciones nunca son previsibles debido a que un grupo muy heterogéneo y diverso. Por todo ello, queremos concluir señalando que el trabajo con personas con discapacidad intelectual es una disciplina compleja, en constante análisis e investigación que merece la pena descubrir.

\section{REFERENCIAS BIBLIOGRÁFICAS}

BISQUERRA, R. (2000) Métodos de investigación educativa guía práctica, Barcelona, CEAC

CASTEJÓN, J.L. y NAVAS, L. (Ed.) (2008) Unas bases psicológicas de la educación especial, Alicante, Editorial Club Universitario

COLL, F.J. (2006) Arteterapia: dinámicas entre creación y procesos terapéuticos, Murcia, Universidad de Murcia

ESCRIBANO, A. (2004) Aprender a enseñar: fundamentos de didáctica general, Cuenca, Universidad de Castilla-La Mancha 
GIMENO, J. y PÉREZ, A. (Eds.) (1989) La enseñanza: su teoría y su práctica, Madrid, Akal

LATORRE, A. (2003) Investigación-acción: conocer y cambiar la práctica educativa, Barcelona, Editorial Grao

LÓPEZ, M. (Coord.) (2006) Creación y Posibilidad: aplicaciones del arte en la integración social, Madrid, Editorial Fundamentos

MORENO, J. M. y MONTERO, P. J. (2007) Intervención educativa en la discapacidad intelectual: talleres y aplicaciones prácticas, Madrid, EOS

VERDUGO, M.A. y CARROBLES, J.A. (1989) La integración personal, social $y$ vocacional de los deficientes psíquicos adolescentes: elaboración y aplicación experimental de un programa conductal, Madrid, CIDE

VV.AA. (2013) Catálogo de la exposición Diálogos, Valladolid, Museo Patio Herreriano y DKV Seguros

WIESNER, J.E. (2004) Discapacidad y capacidad intelectual: en el fulano, el autista, el anciano, el amnésico, el disléxico, el genio-idiota, el lactante y en el paupérrimo, Bogotá, Academia Nacional de Medicina 